\title{
Positive lymph node ratio predicts adverse prognosis for patients with lymph nodes metastatic hypopharyngeal squamous cell carcinoma after primary surgery
}

\author{
Ke Wang", Wendong Tian", Xia Xu, Xiaohong Peng, Haocheng Tang, Yunteng Zhao, Xianwen Wang, \\ Gang $\mathbf{L i}^{\wedge}$ \\ Department of Otolaryngology-Head and Neck Surgery, Nanfang Hospital, Southern Medical University, Guangzhou, China \\ Contributions: (I) Conception and design: K Wang, G Li; (II) Administrative support: G Li; (III) Provision of study materials or patients: K Wang, W \\ Tian; (IV) Collection and assembly of data: X Xu, X Peng; (V) Data analysis and interpretation: H Tang, Y Zhao, X Wang; (VI) Manuscript writing: \\ All authors; (VII) Final approval of manuscript: All authors. \\ \#These authors contributed equally to this work. \\ Correspondence to: Gang Li. Department of Otolaryngology-Head and Neck Surgery, Nanfang Hospital, Southern Medical University, Guangzhou \\ 510515, China. Email: lg@smu.edu.cn.
}

\begin{abstract}
Background: Positive lymph node ratio (LNR) is associated with the prognosis of many cancers. However, its prognostic value in patients with hypopharyngeal squamous cell carcinoma (HSCC) is unclear due to the rarity of HSCC. This study aimed to investigate the prognostic value of LNR in HSCC using the Surveillance, Epidemiology, and End Results (SEER) database.
\end{abstract}

Methods: Data spanning 2004 to 2015 of eligible HSCC patients were retrospectively retrieved from the SEER database. Clinicopathological data, including age at diagnosis, race, gender, marital status, primary tumor site, tumor size, tumor grade, Tumor-Lymph Node-Metastasis (TNM) stage, surgical type, postoperative adjuvant therapy (POAT) record, the number of lymph nodes (LNs) examined, the number of positive LNs, survival time, and death classification were collected and dichotomized through the receiver operating characteristic (ROC) curve. The LNR was defined as the ratio of positive LNs to the total number of LNs examined. The Kaplan-Meier method and Cox regression models were used to assess the association between LNR vs. cancer-specific survival (CSS) and overall survival (OS).

Results: The 5-year CSS and OS rates of the 391 patients were $44 \%$ and $33.7 \%$, respectively. The median LNR was 0.083 [interquartile range (IQR), 0.043-0.179], and the optimal cut-off value of LNR was 0.23. Kaplan-Meier curves showed that patients with LNR $\geq 0.23$ had significantly shorter CSS and OS than LNR <0.23. In multivariable analysis, large tumor size [hazard ratio (HR): 1.012, $\mathrm{P}=0.016$ ], N3 stage (HR: 2.113, $\mathrm{P}=0.040$ ), $\mathrm{M} 1$ stage (HR: 2.458, $\mathrm{P}=0.041$ ), with POAT (HR: 0.559, $\mathrm{P}=0.001$ ), and $\mathrm{LNR} \geq 0.23$ (HR: 1.795, $\mathrm{P}=0.001)$ independently predicted CSS, while old age (HR: 1.019, $\mathrm{P}=0.009)$, large tumor size (HR: 1.012, $\mathrm{P}=0.006$ ), $\mathrm{M} 1$ stage (HR: 3.422, $\mathrm{P}=0.001$ ), with POAT (HR: 0.610, $\mathrm{P}=0.001$ ), and $\mathrm{LNR} \geq 0.23$ (HR: 1.667, $\mathrm{P}=0.001$ ) independently predicted OS. The subgroup analysis showed that patients with $\mathrm{LNR} \geq 0.23$ shared worse CSS and OS in either N2 or N3 subgroups than those with LNR $<0.23$. Furthermore, POAT provided an independent protective factor in the LNR $\geq 0.23$ group, while it had no significant effect in the LNR $<0.23$ group.

Conclusions: This study demonstrates a strong association between LNR and prognosis in patients with LNs metastatic HSCC. Further, it provides an alternative tool for providing supplemental information regarding prognosis.

$\wedge$ ORCID: 0000-0003-3522-3231. 


\begin{abstract}
Keywords: Hypopharyngeal cancer; lymph node ratio (LNR); cancer-specific survival (CSS); overall survival (OS);
\end{abstract} postoperative adjuvant therapy (POAT)

Submitted Aug 25, 2021. Accepted for publication Jan 27, 2022.

doi: $10.21037 /$ tcr-21-1740

View this article at: https://dx.doi.org/10.21037/tcr-21-1740

\section{Introduction}

Hypopharyngeal squamous cell carcinoma (HSCC) is a rare malignant tumor, accounting for about $2-6 \%$ of head and neck cancers (1). However, the prognosis of HSCC patients is poor, with an estimated 5-year overall survival rate (OS) of about $30-35 \%(2,3)$. The rich lymph network near the cancer area and the submucosal extension promotes LNs metastasis, thus leading to an extremely low survival rate. Previous studies have shown that almost $60-70 \%$ of HSCC patients have lymph nodes (LNs) metastasis at diagnosis. Moreover, the risk of recurrence in LNs metastatic HSCC is high $(4,5)$. Nodal staging for HSCC patients is primarily based on the size and laterality of positive LNs, with less value placed on the absolute number of positive LNs and surgically-removed LNs (6).

Most studies have analyzed the relationship between positive LNs and prognosis in HSCC patients (7-9). Moreover, the number of positive LNs is closely related to the number of LNs removed during surgery. However, the burden of positive LNs cannot reflect the true status of the nodal condition if there is a limited number of LNs dissected. Besides, the number of LNs removed during surgery can significantly affect prognosis in HSCC patients $(10,11)$. In recent years, positive lymph node ratio (LNR) has been used as an independent prognostic factor in many malignancies (12-16). Besides the information on the burden of LNs metastasis, it infers the number of LNs dissected during surgery. Furthermore, recent studies have suggested that LNR is better than the TumorLymph Node-Metastasis (TNM) staging and the absolute number of positive LNs in predicting the postoperative cancer-specific survival (CSS) rate of patients with bladder, laryngeal, and lung cancers $(14,17,18)$. The HSCC patients with higher LNR are associated with worse survival (19-22). However, those studies were single-center analyses with limited sample sizes ( 41-121 patients). Besides, the studies had an unknown number of LNs harvested, and those with an insufficient number of LNs harvested during surgery could have caused risk overestimation. As a result, this study aimed to investigate the prognostic value of LNR in patients with LNs metastatic HSCC after primary surgery. We present the following article in accordance with the STROBE reporting checklist (available at https://tcr. amegroups.com/article/view/10.21037/tcr-21-1740/rc).

\section{Methods}

\section{Study cobort}

The study was a retrospective analysis of HSCC patients based on data from the Surveillance, Epidemiology, and End Results (SEER) database, containing about $28 \%$ of the U.S. population. The study was conducted in accordance with the Declaration of Helsinki (as revised in 2013). Detailed descriptions of the data were obtained from the official SEER website (https://seer.cancer.gov/data/). A total of 6,798 hypopharynx patients (TNM 7/CS v0204+ Schema = 'Hypopharynx') were obtained from January 2004 to December 2015. Inclusion criteria were: (I) patients who underwent primary surgery; (II) patients with pathologically confirmed HSCC; and (III) patients with positive LNs examined. Exclusion criteria were: (I) patients with $<10 \mathrm{LNs}$ examined; (II) patients with unknown number of positive LNs or LNs examined; (III) patients who survived less than three months after surgery; (IV) patients with incomplete clinic and prognostic data. The inclusion and exclusion flowchart is shown in Figure 1.

\section{Data collection}

The age at diagnosis, race (recorded as "white" or "others"), gender, marital status ("unmarried" or "married"), primary tumor site (recorded as "pyriform sinus" or "others"), tumor size, tumor grade, TNM stage ( $6^{\text {th }}$ edition), surgical type (recorded as "local tumor resection", "pharyngectomy", or "pharyngectomy with laryngectomy"), radiotherapy record, chemotherapy record, the number of LNs examined, the number of positive LNs, survival time, and death classification, were retrieved for further analysis. Patients who received either chemotherapy or radiotherapy were recorded as "with POAT". Otherwise, they were recorded 


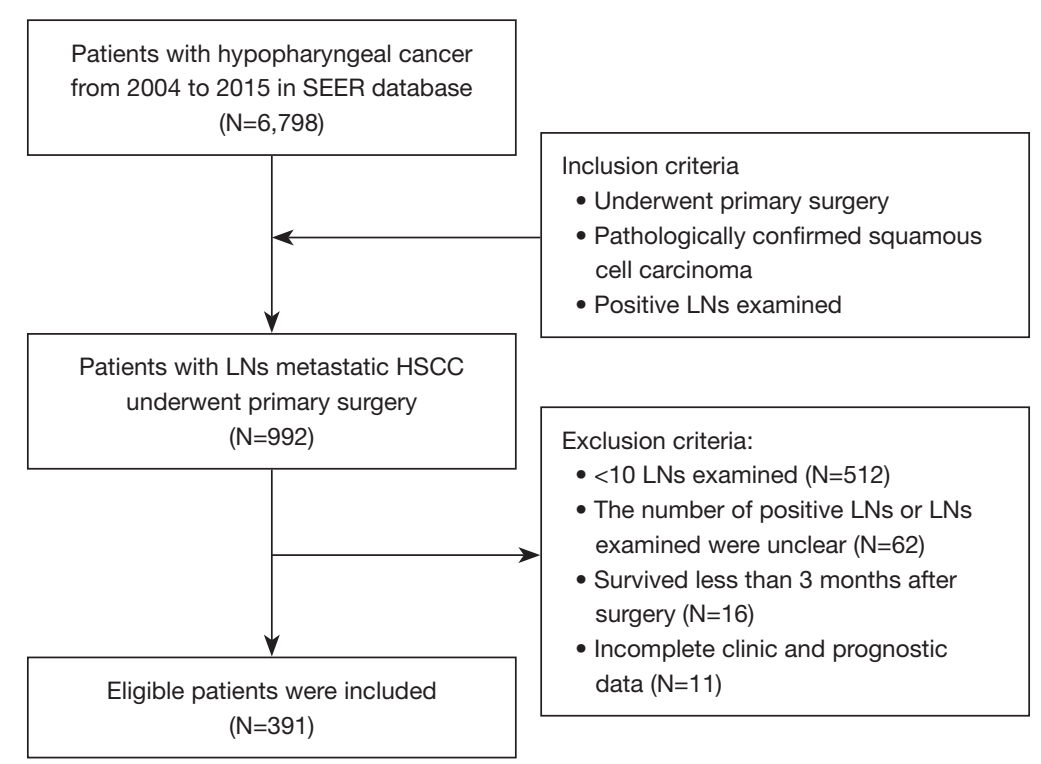

Figure 1 Summary of our study cohort and flow chart of inclusion and exclusion criteria. HSCC, hypopharyngeal squamous cell carcinoma; LN, lymph node; SEER, Surveillance, Epidemiology, and End Results.

as "without POAT". LNR (positive LNs density) was defined as the ratio of the number of positive LNs to the total number of LNs examined.

CSS was identified as the primary endpoint, and it was defined as the period from the date of diagnosis to cancer-specific death. The OS was regarded as the second endpoint, and it was defined as the period from the date of diagnosis to death of all causes.

\section{Statistical analysis}

The receiver operating characteristic (ROC) curve associated with cancer-specific death was used to determine the optimal cut-off value of LNR. This study also compared the clinicopathological characteristics of low and high LNR. The normally distributed continuous variables were recorded as mean and standard deviation (SD) and compared using student's $t$-test. Otherwise, they were reported as medians and interquartile range (IQR) and compared using Mann-Whitney $U$ test. Categorical variables were expressed as frequency count and percentage and compared using the Chi-square test. The Kaplan-Meier method was used to estimate the CSS and OS and compared using log rank-test. The univariate Cox proportional hazards regression model was used to assess the association between LNR vs. CSS and OS. Multivariate Cox proportional hazards regression model was used to assess the prognostic value of LNR adjusted for other clinicopathological characteristics. The results were summarized using a hazard ratio (HR) and 95\% confidence interval (CI). The univariate and multivariate Cox proportional hazards regression models were used to analyze the interaction effect of the LNR group and clinicopathological factors on CSS and OS.

Statistical analysis was performed using SPSS version 22 (IBM Corp, Armonk, NY, USA) and plotting done in GraphPad Prism version 8 (GraphPad Software Inc., San Diego, CA, USA). All tests were two-sided, and $\mathrm{P}<0.05$ was considered statistically significant.

\section{Results}

\section{Clinicopatbological characteristics}

This study identified 391 patients with LNs metastatic HSCC [median age: 61 years (IQR, 55-69 years), and 330 (84.4\%) patients were males]. All patients had received primary surgery and neck LNs dissection. The medians of the examined LNs, positive LNs, and LNR were 37 (IQR, 23-55), 3 (IQR, 1-6), and 0.083 (IQR, 0.043-0.179), respectively. Most patients $(\mathrm{N}=312,79.8 \%)$ received postoperative adjuvant therapy (POAT) after surgery. The Median follow-up duration was 30 months (IQR, 15-59 months). A total of 218 (55.8\%) cancer-specific deaths with estimated 3- and 5-year CSS rates of 52.7\% 
and $44 \%$, respectively, were reported during the follow-up. Besides, 292 (74.7\%) all-cause deaths with estimated 3- and 5 -year OS rates of $45.3 \%$ and $33.7 \%$, respectively, were reported.

This study selected a cut-off value at 0.23 using the ROC curve area and the Youden Index for dichotomization since there is no widely recognized cut-off value for LNR. Patients with $\mathrm{LNR} \geq 0.23$ were associated with advanced $\mathrm{N}$ and $\mathrm{M}$ stages than LNR $<0.23$ (Table 1). However, other clinicopathological parameters were similar between the two groups. Besides, patients with $L N R \geq 0.23$ had significantly shorter CSS and OS than those with LNR $<0.23(\mathrm{P}<0.001$, Figure 2).

\section{Prognostic predictors}

The univariable and multivariable Cox regression models predicting CSS for HSCC patents are shown in Table 2. The tumor size (HR: 1.013, $\mathrm{P}=0.001$ ), advanced $\mathrm{T}$ stage (HR: $1.559, \mathrm{P}=0.004)$, advanced $\mathrm{N}$ stage $(\mathrm{N} 2, \mathrm{HR}: 1.536$, $\mathrm{P}=0.021$; N3, HR: 2.505, $\mathrm{P}=0.003$ ), M1 stage (HR: 2.512, $\mathrm{P}=0.018$ ), POAT (HR: 0.699, $\mathrm{P}=0.029$ ), and $\mathrm{LNR} \geq 0.23$ (HR: 2.037, $\mathrm{P}<0.001$ ) in the univariable model were associated with CSS. Moreover, large tumor size (HR: 1.012, $\mathrm{P}=0.016$ ), $\mathrm{N} 3$ stage (HR: 2.113, $\mathrm{P}=0.040), \mathrm{M} 1$ stage (HR: 2.458, $\mathrm{P}=0.041$ ), with POAT (HR: 0.559, $\mathrm{P}=0.001$ ), and $\mathrm{LNR} \geq 0.23$ (HR: $1.795, \mathrm{P}=0.001$ ) in the multivariable model independently predicted CSS. Besides, age (HR: $1.020, \mathrm{P}=0.003$ ), tumor size (HR: $1.011, \mathrm{P}=0.002$ ), advanced $T$ stage (HR: $1.342, \mathrm{P}=0.021), \mathrm{M} 1$ stage (HR: 3.060, $\mathrm{P}=0.001$ ), surgical type (HR: $1.468, \mathrm{P}=0.031$ ), with POAT (HR: 0.686, $\mathrm{P}=0.008$ ), and $\mathrm{LNR} \geq 0.23$ (HR: 1.820, $\mathrm{P}<0.001)$ in the univariable model were associated with OS (Table 3). The old age (HR: 1.019, $\mathrm{P}=0.009)$, large tumor size (HR: 1.012, $\mathrm{P}=0.006)$, $\mathrm{M} 1$ stage (HR: 3.422, $\mathrm{P}=0.001$ ), with POAT (HR: 0.610, $\mathrm{P}=0.001$ ), and $\mathrm{LNR} \geq 0.23$ (HR: $1.667, \mathrm{P}=0.001$ ) in the multivariable model independently predicted OS. This study set the cut-off point of LNR using a quartile to validate the prognostic value of LNR further. Patients with $\mathrm{LNR} \geq 0.179$ in the Cox regression model independently predicted worse CSS (HR: 1.531, $\mathrm{P}=0.013$ ) and OS (HR: 1.392, $\mathrm{P}=0.024)$.

\section{The value of LNR in different subgroups}

The subgroup analysis showed that $\mathrm{N} 2$ patients with LNR $<0.23$ had a better CSS and OS than those with LNR $\geq 0.23$ $(\mathrm{P}=0.004$ and 0.002 , respectively, Figure $3 A, 3 B)$. Similarly, the N3 patients with LNR $<0.23$ had a better CSS and OS than those with $\mathrm{LNR} \geq 0.23$ ( $\mathrm{P}=0.027$ and 0.043 , respectively, Figure 3C,3D). This study further compared "with POAT" and "without POAT" in LNR $<0.23$ and LNR $\geq 0.23$ groups to determine the value of POAT for patients with different LNR subgroups. Patients with and without POAT had similar CSS and OS in the LNR $<0.23$ group $(\mathrm{P}=0.235$ and 0.092, respectively, Figure $4 A, 4 B)$. However, patients with POAT had significantly better CSS and OS than those without POAT in the $\mathrm{LNR} \geq 0.23$ group $(\mathrm{P}=0.004$ and 0.002 , respectively, Figure $4 C, 4 D$ ). The multivariate analysis showed that POAT was an independent protective factor for CSS (HR: 0.489, $\mathrm{P}=0.037$ ) and OS (HR: 0.459, $\mathrm{P}=0.016$ ) in patients with LNR $\geq 0.23$ group. However, POAT had no significant effect on CSS and OS in patients with LNR $<0.23$ (Tables S1,S2).

\section{Discussion}

The study investigated the relationship between LNR and prognosis of LNs metastatic HSCC patients using SEER databases. The results showed that patients with $\mathrm{LNR} \geq 0.23$ were associated with advanced $\mathrm{N}$ and $\mathrm{M}$ stages and shorter CSS and OS than patients with LNR $<0.23$. Multivariable analysis showed that besides age, tumor size, TNM stage, and POAT, LNR was an independent predictor of CSS and OS. Subgroup analysis showed that N2 and N3 patients with LNR $<0.23$ had a better prognosis than those with LNR $>0.23$. Furthermore, POAT was an independent protective factor for patients with $\mathrm{LNR} \geq 0.23$, while it had no significant effect in patients with $\mathrm{LNR}<0.23$.

Currently, Tumor-Lymph Node-Metastasis (TNM) staging is the most commonly used prognostic model for HSCC patients. The nodal stage is based on the number, size, and laterality of positive neck LNs (6). However, it does not evaluate LNs metastasis burden. Several studies have reported that the absolute number of positive $\mathrm{LNs}$ is associated with the prognosis for HSCC patients $(7,8)$. For instance, Choi et al. (8) investigated 141 consecutive patients with HSCC and found that the number of positive LNs was strongly associated with disease-free survival (DFS) and OS outcomes $(\mathrm{P}<0.01)$. Ho et al. (7) also retrospectively analyzed 8,351 cases (largest series) and found that mortality risk increases as the number of metastatic LNs increases, with the hazard per node (HR: 1.19; 95\% CI: 1.16-1.23; $\mathrm{P}<0.001)$ being most pronounced up to five positive LNs. Besides, they proposed a novel nodal stage, which exhibited greater concordance with survival than the TNM staging 
Table 1 Demographics and characteristics of patients separated by lymph node ratio

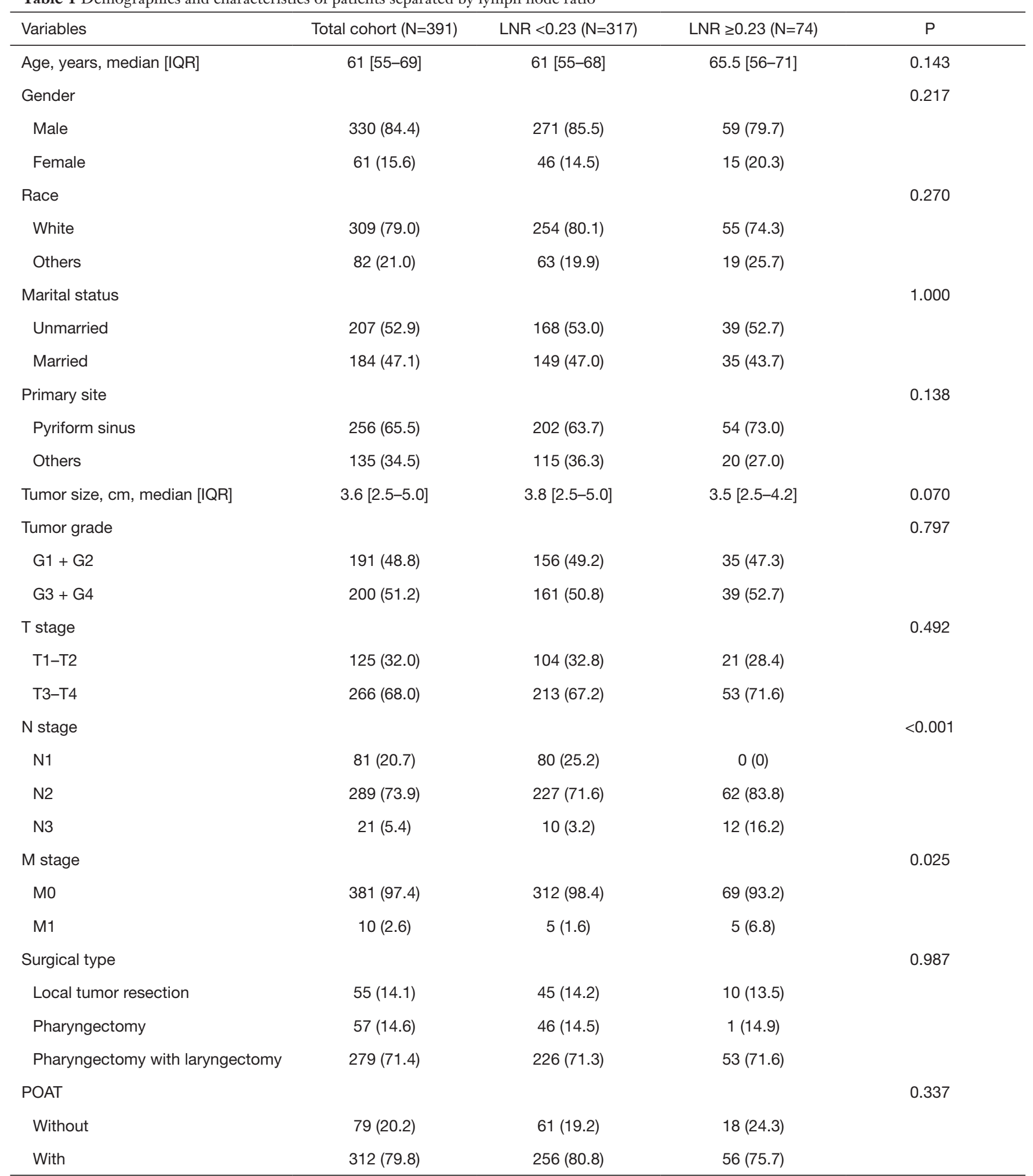

IQR, interquartile range; LNR, lymph node ratio; POAT, postoperative adjuvant therapy. 
A

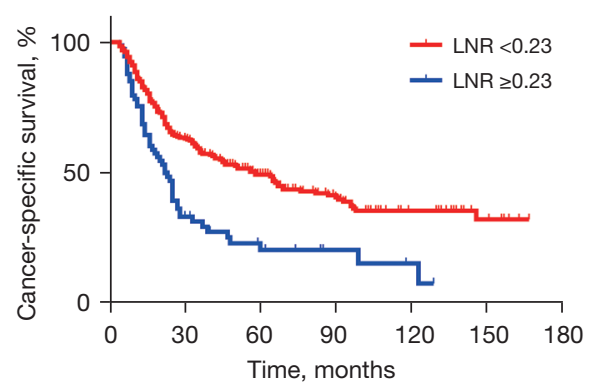

B

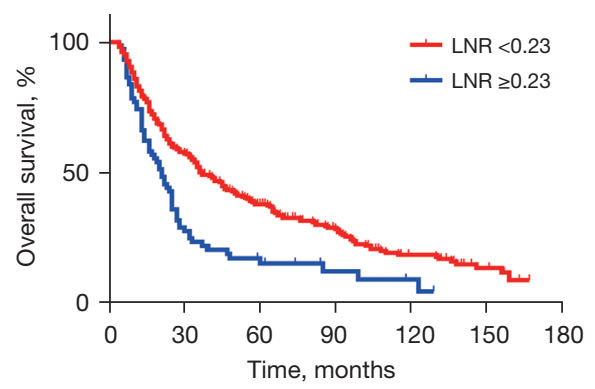

Figure 2 Kaplan-Meier curve demonstrating cancer-specific survival (CSS) and overall survival (OS) for our study cohort separated by LNR $<0.23$ and LNR $\geq 0.23$. Survival curves for: (A) CSS, log-rank $\mathrm{P}<0.001$; (B) OS, log-rank $\mathrm{P}<0.001$. LNR, positive lymph node ratio.

Table 2 Univariable and multivariable Cox regression model for predictors of cancer-specific survival in patients with lymph nodes metastatic hypopharyngeal squamous cell carcinoma after primary surgery

\begin{tabular}{|c|c|c|c|c|c|c|}
\hline Variables & \multicolumn{3}{|c|}{ Univariable analysis } & \multicolumn{3}{|c|}{ Multivariable analysis } \\
\hline Age, years & 1.015 & $0.999-1.030$ & 0.064 & & & \\
\hline \multicolumn{7}{|l|}{ Gender } \\
\hline Male & Ref. & & & & & \\
\hline \multicolumn{7}{|l|}{ Race } \\
\hline White & Ref. & & & & & \\
\hline Others & 1.117 & $0.806-1.548$ & 0.506 & & & \\
\hline \multicolumn{7}{|l|}{ Marital status } \\
\hline \multicolumn{7}{|l|}{ Primary site } \\
\hline Pyriform sinus & Ref. & & & & & \\
\hline Others & 1.011 & $0.764-1.339$ & 0.938 & & & \\
\hline Tumor size, cm & 1.013 & $1.006-1.021$ & 0.001 & 1.012 & $1.002-1.022$ & 0.016 \\
\hline \multicolumn{7}{|l|}{ Tumor grade } \\
\hline $\mathrm{G} 1+\mathrm{G} 2$ & Ref. & & & & & \\
\hline $\mathrm{G} 3+\mathrm{G} 4$ & 0.917 & $0.703-1.196$ & 0.522 & & & \\
\hline \multicolumn{7}{|l|}{ T stage } \\
\hline
\end{tabular}

Table 2 (continued) 
Table 2 (continued)

\begin{tabular}{|c|c|c|c|c|c|c|}
\hline Variables & \multicolumn{3}{|c|}{ Univariable analysis } & \multicolumn{3}{|c|}{ Multivariable analysis } \\
\hline \multicolumn{7}{|l|}{$\mathrm{N}$ stage } \\
\hline $\mathrm{N} 1$ & Ref. & & & Ref. & & \\
\hline N2 & 1.536 & $1.067-2.212$ & 0.021 & 1.500 & $0.992-2.267$ & 0.055 \\
\hline \multicolumn{7}{|l|}{ M stage } \\
\hline MO & Ref. & & & Ref. & & \\
\hline M1 & 2.512 & $1.174-5.373$ & 0.018 & 2.458 & $1.039-5.815$ & 0.041 \\
\hline \multicolumn{7}{|l|}{ Surgical type } \\
\hline Pharyngectomy with laryngectomy & 1.495 & $0.995-2.246$ & 0.053 & & & \\
\hline \multicolumn{7}{|l|}{ POAT } \\
\hline Without & Ref. & & & Ref. & & \\
\hline With & 0.699 & $0.507-0.963$ & 0.029 & 0.559 & $0.394-0.793$ & 0.001 \\
\hline \multicolumn{7}{|l|}{ LNR (dichotomized by ROC) } \\
\hline$<0.23$ & Ref. & & & Ref. & & \\
\hline$\geq 0.23$ & 2.037 & $1.497-2.773$ & $<0.001$ & 1.795 & $1.256-2.565$ & 0.001 \\
\hline \multicolumn{7}{|l|}{ LNR (dichotomized by quartiles)* } \\
\hline
\end{tabular}

*, separate model with the inclusion of LNR (dichotomized by quartiles) and exclusion of LNR (dichotomized by ROC). Cl, confidence interval; HR, hazard ratio; LNR, lymph node ratio; POAT, postoperative adjuvant therapy; ROC, receiver operating characteristic.

system. To the best of our knowledge, the scope of LNs dissection and the number of LNs harvested during surgery can also significantly affect the number of positive LNs. Furthermore, researchers have also found that the number of LNs dissected can affect prognosis even in patients with negative LNs, suggesting that the burden of metastatic LNs and the surgical performance (the number of LNs removed) affect HSCC prognosis (10). Therefore, LNR may have a greater prognostic value than the absolute number of positive LNs since it involves two factors, the number of positive LNs and the extent of surgical treatment (number of LNs removed).

High LNR is associated with adverse prognosis in many malignancies (12-18). Moreover, Suzuki et al. (19) demonstrated that LNR is a prognostic factor for HSCC patients with positive LNs. However, the study had a small sample size of only 46 patients, and thus further studies are necessary. Hua et al. (20) also analyzed 81 patients from a single center and found that $\mathrm{LNR}<0.1$ is associated with significantly longer survival than $\mathrm{LNR}>0.1$, even in the $\mathrm{N} 1$ and N2 subgroups. Two studies from South Korea also showed high LNR can independently predict adverse prognosis for HSCC patients $(21,22)$. This study also suggested that high LNR can independently predict CSS and OS. This study also found that LNR could conduct further risk stratification for patients in the same N2 or N3 stages. Although high LNR was correlated with poor prognosis, the cut-off value was different across various studies. For instance, in the Suzuki et al. (19) and Hua et al. (20), the cut-off value of LNR were 0.09 and 0.1 , 
Table 3 Univariable and multivariable Cox regression model for predictors of overall survival in patients with lymph nodes metastatic hypopharyngeal squamous cell carcinoma after primary surgery

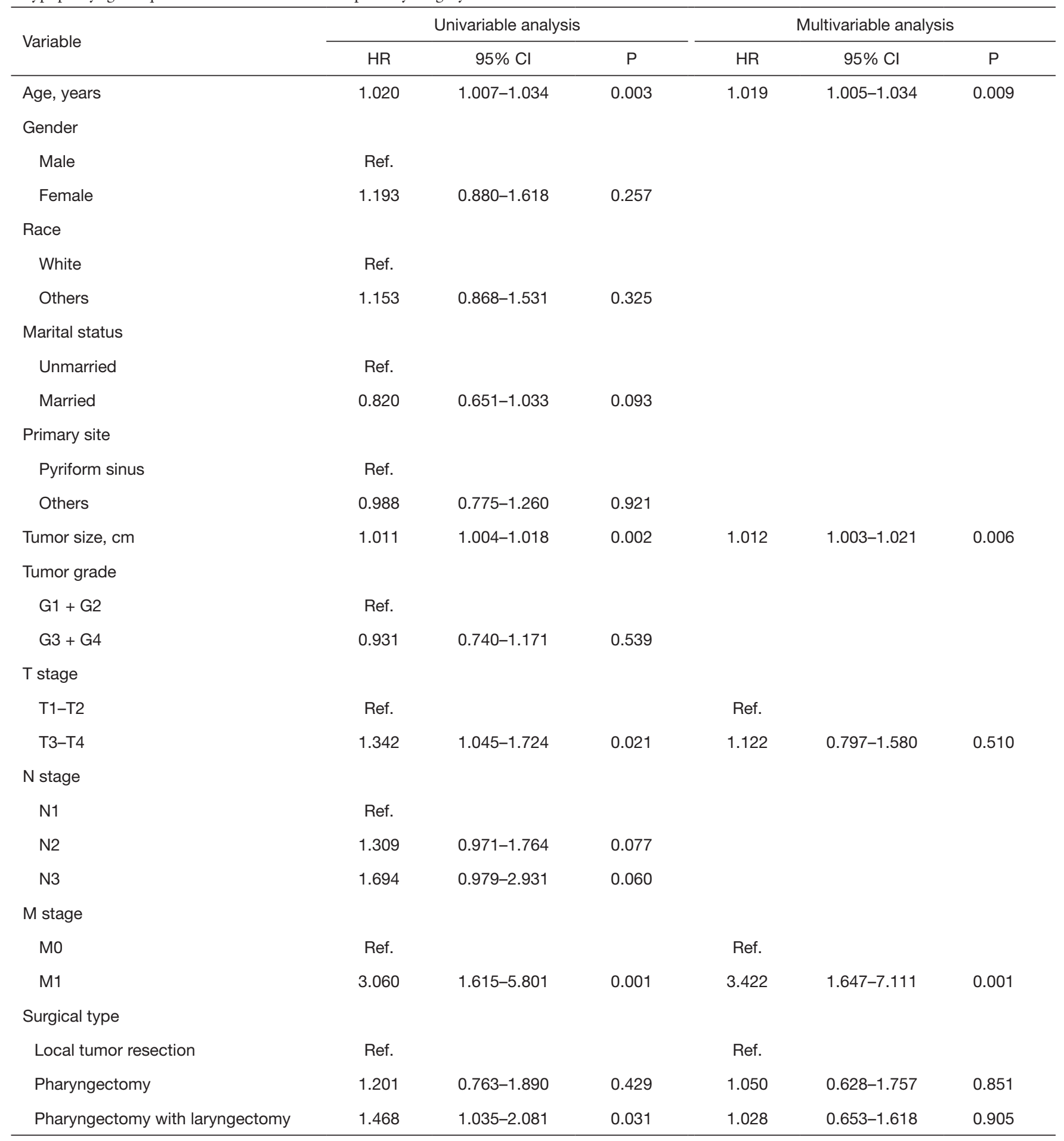

Table 3 (continued) 
Table 3 (continued)

\begin{tabular}{|c|c|c|c|c|c|c|}
\hline Variable & \multicolumn{3}{|c|}{ Univariable analysis } & \multicolumn{3}{|c|}{ Multivariable analysis } \\
\hline \multicolumn{7}{|l|}{ POAT } \\
\hline Without & Ref. & & & Ref. & & \\
\hline With & 0.686 & $0.520-0.906$ & 0.008 & 0.610 & $0.450-0.826$ & 0.001 \\
\hline$<0.23$ & Ref. & & & Ref. & & \\
\hline$\geq 0.23$ & 1.820 & $1.374-2.410$ & $<0.001$ & 1.667 & $1.218-2.283$ & 0.001 \\
\hline \multicolumn{7}{|c|}{ LNR (dichotomized by quartiles) ${ }^{*}$} \\
\hline$<0.179$ & Ref. & & & Ref. & & \\
\hline
\end{tabular}

*, separate model with the inclusion of LNR (dichotomized by quartiles) and exclusion of LNR (dichotomized by ROC). Cl, confidence interval; HR, hazard ratio; LNR, lymph node ratio; POAT, postoperative adjuvant therapy; ROC, receiver operating characteristic.

A

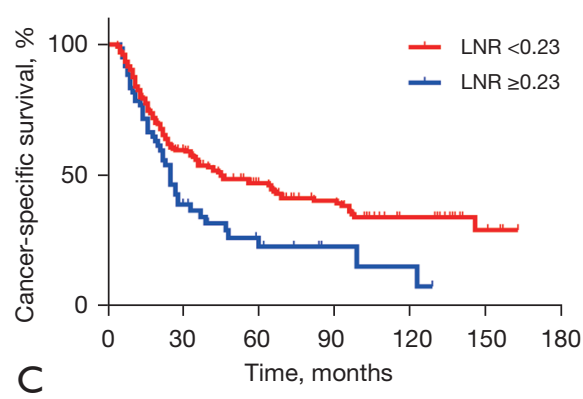

C

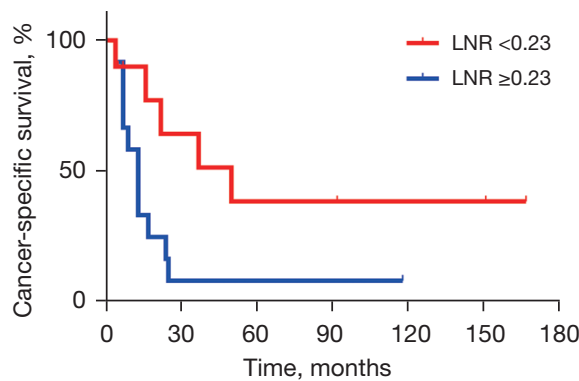

B

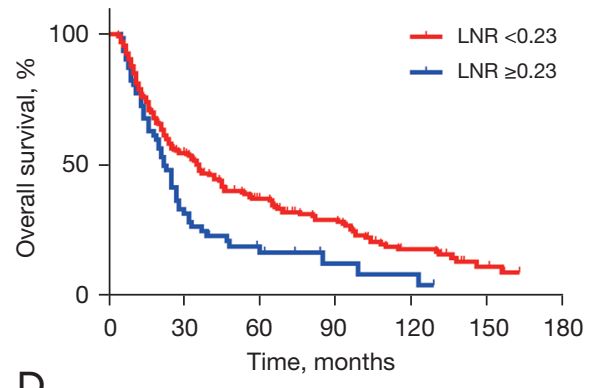

D

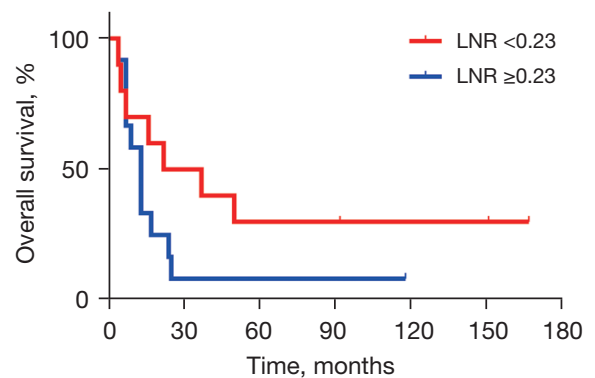

Figure 3 Kaplan-Meier curve demonstrating cancer-specific survival (CSS) and overall survival (OS) for N2 and N3 patients separated by LNR <0.23 and LNR $\geq 0.23$. Survival curves for: (A) CSS in N2 patients, log-rank $\mathrm{P}=0.004$; (B) OS in N2 patients, log-rank $\mathrm{P}=0.002$; (C) CSS in N3 patients, log-rank $\mathrm{P}=0.027$; (D) OS in N3 patients, log-rank $\mathrm{P}=0.043$. LNR, positive lymph node ratio.

respectively, which is relatively lower than that in this study. The difference could be due to the different inclusion criteria among various studies. For instance, this study excluded patients with negative LNs and those with less than 10 LNs examined. It only focused on the positive LNs population with sufficient LNs dissected. Therefore, LNR can provide supplemental information for the prognosis of patients with positive LNs. 

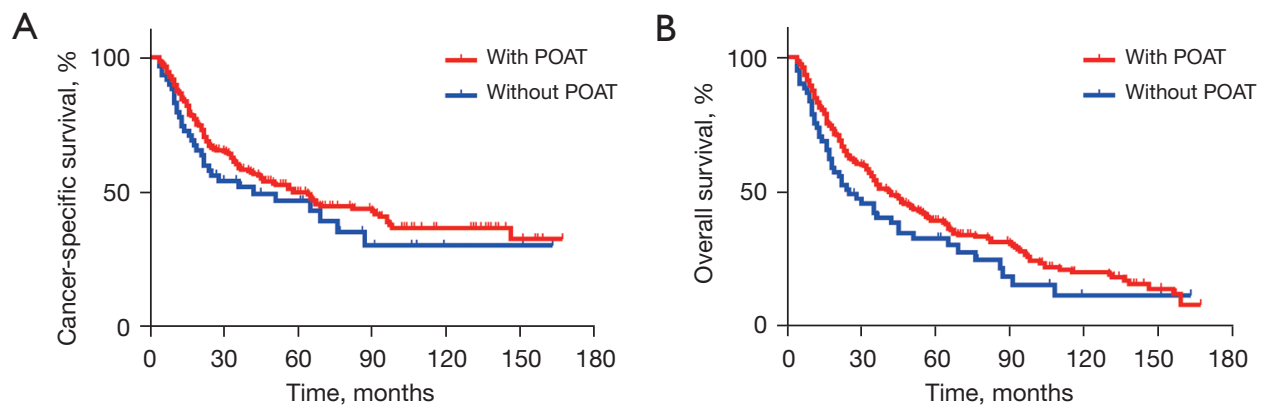

C

$\mathrm{D}$
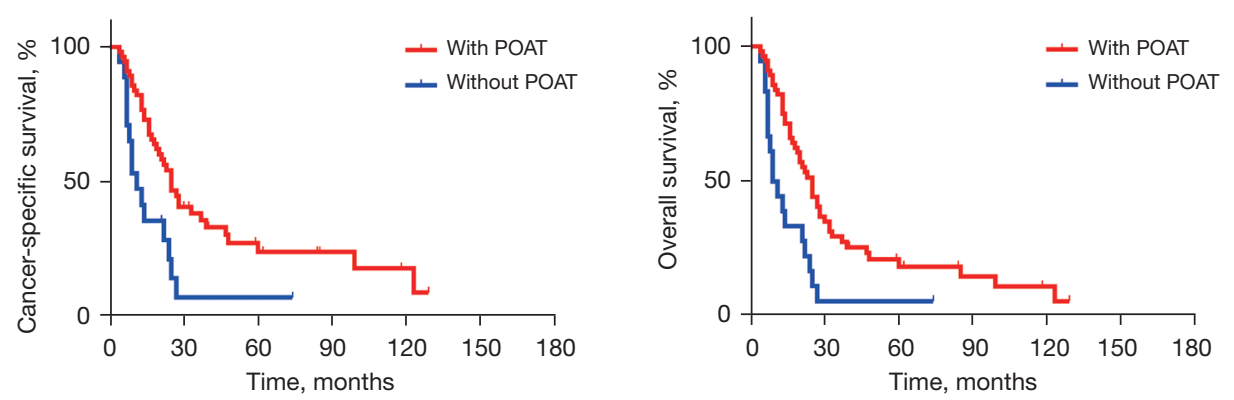

Figure 4 Kaplan-Meier curve demonstrating cancer-specific survival (CSS) and overall survival (OS) for LNR $<0.23$ and LNR $\geq 0.23$ patients separated by postoperative adjuvant therapy (POAT). Survival curves for: (A) CSS in LNR $<0.23$ patients, log-rank $\mathrm{P}=0.235$; (B) OS in LNR <0.23 patients, log-rank $\mathrm{P}=0.092$; (C) CSS in LNR $\geq 0.23$ patients, log-rank $\mathrm{P}=0.004$; (D) OS in LNR $\geq 0.23$ patients, log-rank $\mathrm{P}=0.002$. LNR, positive lymph node ratio.

The optimal treatment modalities for HSCC patients are unknown due to the poor oncologic outcomes. Moreover, non-surgical treatment, including definitive chemoradiotherapy (CRT) and radiotherapy after induction chemotherapy (RT), has been used to treat HSCC patients in recent years (3). Surgery combined with POAT is the optimal choice for patients in the advanced stage (23). However, POAT can severely damage the normal tissue, and thus generating different degrees of radiation-induced cutaneous and mucous reactions $(24,25)$. Therefore, highrisk patients should be identified before POAT. The NCCN guidelines indicate that POAT can treat patients with a positive margin and extranodal extension. However, recent evidence has shown that margin status and extranodal extension cannot identify patients suitable for POAT (9). Besides, stratification via metastatic $\mathrm{LNs}$ numbers can characterize a very high-risk patient cohort suitable for POAT (9). Herein, LNR was used to stratify patients' risk, and the results showed that those who received POAT had a significantly better survival outcome than those without POAT for the LNR $\geq 0.23$ subgroup. However, the patients with or without POAT had a similar survival outcome in the LNR $<0.23$ subgroup. Another research also showed that
POAT can benefit patients with high LNR (26). Therefore, LNR can help clinicians decide which patients could benefit from POAT among patients with LNs positive.

However, this study has some limitations. First, LNR was substantially affected by the quality of neck dissection achieved by the surgeon and the quantity of LNs harvested and examined by the pathologist. The above data could not be unified in the SEER database, thus generating information bias. However, this study excluded the patients with less than $10 \mathrm{LNs}$ examined to ensure the quality of neck dissection and minimize the information bias. Second, the SEER database lacked the information on POAT and thus could affect the prognosis for HSCC patients. The SEER database did not also have the follow-up scheme, thus leading to potential bias. Third, TNM stage data in the SEER database are limited to only the $6^{\text {th }}$ edition from 2004 to 2015 , and thus the latest $8^{\text {th }}$ edition TNM stage manual, commonly used in current clinical practice, could not be obtained.

\section{Conclusions}

In conclusion, this study showed that LNR is associated with the prognosis in patients with LNs metastatic 
HSCC, providing supplemental information on prognosis. However, more prospective studies are needed to confirm the prognostic role of LNR in HSCC.

\section{Acknowledgments}

Funding: None.

\section{Footnote}

Reporting Checklist: The authors have completed the STROBE reporting checklist. Available at https://tcr. amegroups.com/article/view/10.21037/tcr-21-1740/rc

Peer Review File: Available at https://tcr.amegroups.com/ article/view/10.21037/tcr-21-1740/prf

Conflicts of Interest: All authors have completed the ICMJE uniform disclosure form (available at https://tcr.amegroups. com/article/view/10.21037/tcr-21-1740/coif). The authors have no conflicts of interest to declare.

Ethical Statement: The authors are accountable for all aspects of the work in ensuring that questions related to the accuracy or integrity of any part of the work are appropriately investigated and resolved. The study was conducted in accordance with the Declaration of Helsinki (as revised in 2013).

Open Access Statement: This is an Open Access article distributed in accordance with the Creative Commons Attribution-NonCommercial-NoDerivs 4.0 International License (CC BY-NC-ND 4.0), which permits the noncommercial replication and distribution of the article with the strict proviso that no changes or edits are made and the original work is properly cited (including links to both the formal publication through the relevant DOI and the license). See: https://creativecommons.org/licenses/by-nc-nd/4.0/.

\section{References}

1. Siegel RL, Miller KD, Fuchs HE, et al. Cancer Statistics, 2021. CA Cancer J Clin 2021;71:7-33.

2. Newman JR, Connolly TM, Illing EA, et al. Survival trends in hypopharyngeal cancer: a population-based review. Laryngoscope 2015;125:624-9.

3. Takes RP, Strojan P, Silver CE, et al. Current trends in initial management of hypopharyngeal cancer: the declining use of open surgery. Head Neck 2012;34:270-81.

4. Leemans CR, Tiwari R, Nauta JJ, et al. Regional lymph node involvement and its significance in the development of distant metastases in head and neck carcinoma. Cancer 1993;71:452-6.

5. Layland MK, Sessions DG, Lenox J. The influence of lymph node metastasis in the treatment of squamous cell carcinoma of the oral cavity, oropharynx, larynx, and hypopharynx: $\mathrm{N} 0$ versus $\mathrm{N}+$. Laryngoscope 2005;115:629-39.

6. Amin MB, Edge S, Greene F, et al. The 8th edition of the AJCC Cancer Staging Manual. Springer, 2017.

7. Ho AS, Kim S, Tighiouart M, et al. Association of Quantitative Metastatic Lymph Node Burden With Survival in Hypopharyngeal and Laryngeal Cancer. JAMA Oncol 2018;4:985-9.

8. Choi Y, Bin-Manie M, Roh JL, et al. Metastatic lymph node burden predictive of survival in patients undergoing primary surgery for laryngeal and hypopharyngeal cancer. J Cancer Res Clin Oncol 2019;145:2565-72.

9. Zumsteg ZS, Luu M, Kim S, et al. Quantitative lymph node burden as a 'very-high-risk' factor identifying head and neck cancer patients benefiting from postoperative chemoradiation. Ann Oncol 2019;30:76-84.

10. Divi V, Chen MM, Nussenbaum B, et al. Lymph Node Count From Neck Dissection Predicts Mortality in Head and Neck Cancer. J Clin Oncol 2016;34:3892-7.

11. León X, Venegas MDP, Casasayas M, et al. Prognostic value of the nodal yield in elective neck dissections in patients with head and neck carcinomas. Eur Arch Otorhinolaryngol 2022;279:883-9.

12. Ebrahimi A, Clark JR, Zhang WJ, et al. Lymph node ratio as an independent prognostic factor in oral squamous cell carcinoma. Head Neck 2011;33:1245-51.

13. Erstad DJ, Blum M, Estrella JS, et al. Benchmarks for nodal yield and ratio for node-positive gastric cancer. Surgery 2021;170:1231-9.

14. Li F, Yuan L, Zhao Y, et al. Comparison of Two Proposed Changes to the Current Nodal Classification for Non-small Cell Lung Cancer Based on the Number and Ratio of Metastatic Lymph Nodes. Chest 2021;160:1520-33.

15. Detering R, Meyer VM, Borstlap WAA, et al. Prognostic importance of lymph node count and ratio in rectal cancer after neoadjuvant chemoradiotherapy: Results from a cross-sectional study. J Surg Oncol 2021;124:367-77.

16. Xu T, Zhang $\mathrm{L}$, Yu L, et al. Log odds of positive lymph nodes is an excellent prognostic factor for patients with rectal cancer after neoadjuvant chemoradiotherapy. Ann 
Transl Med 2021;9:637.

17. Kassouf W, Agarwal PK, Herr HW, et al. Lymph node density is superior to TNM nodal status in predicting disease-specific survival after radical cystectomy for bladder cancer: analysis of pooled data from MDACC and MSKCC. J Clin Oncol 2008;26:121-6.

18. Ryu IS, Roh JL, Cho KJ, et al. Lymph node density as an independent predictor of cancer-specific mortality in patients with lymph node-positive laryngeal squamous cell carcinoma after laryngectomy. Head Neck 2015;37:1319-25.

19. Suzuki H, Matoba T, Hanai N, et al. Lymph node ratio predicts survival in hypopharyngeal cancer with positive lymph node metastasis. Eur Arch Otorhinolaryngol 2016;273:4595-600.

20. Hua YH, Hu QY, Piao YF, et al. Effect of number and ratio of positive lymph nodes in hypopharyngeal cancer. Head Neck 2015;37:111-6.

21. Lo WC, Wu CT, Wang CP, et al. Lymph Node Ratio Predicts Recurrence and Survival for Patients with Resectable Stage 4 Hypopharyngeal Cancer. Ann Surg

Cite this article as: Wang $\mathrm{K}$, Tian $\mathrm{W}$, Xu X, Peng X, Tang H, Zhao Y, Wang X, Li G. Positive lymph node ratio predicts adverse prognosis for patients with lymph nodes metastatic hypopharyngeal squamous cell carcinoma after primary surgery. Transl Cancer Res 2022;11(3):463-474. doi: 10.21037/tcr-21-1740
Oncol 2017;24:1707-13.

22. Joo YH, Cho KJ, Kim SY, et al. Prognostic Significance of Lymph Node Density in Patients with Hypopharyngeal Squamous Cell Carcinoma. Ann Surg Oncol 2015;22 Suppl 3:S1014-9.

23. Harris BN, Biron VL, Donald P, et al. Primary Surgery vs Chemoradiation Treatment of Advanced-Stage Hypopharyngeal Squamous Cell Carcinoma. JAMA Otolaryngol Head Neck Surg 2015;141:636-40.

24. Lee NY, O'Meara W, Chan K, et al. Concurrent chemotherapy and intensity-modulated radiotherapy for locoregionally advanced laryngeal and hypopharyngeal cancers. Int J Radiat Oncol Biol Phys 2007;69:459-68.

25. Tombolini V, Santarelli M, Raffetto N, et al. Radiotherapy in the treatment of stage III-IV hypopharyngeal carcinoma. Anticancer Res 2004;24:349-54.

26. Wang YL, Feng SH, Zhu J, et al. Impact of lymph node ratio on the survival of patients with hypopharyngeal squamous cell carcinoma: a population-based analysis. PLoS One 2013;8:e56613. 
Supplementary

Table S1 Univariable and multivariable Cox regression model for predictors of cancer-specific survival in patients with LNR $<0.23$ and LNR $\geq 0.23$

\begin{tabular}{|c|c|c|c|c|c|c|c|c|}
\hline \multirow{3}{*}{ Variable } & \multicolumn{4}{|c|}{ LNR $<0.23$} & \multicolumn{4}{|c|}{$L N R \geq 0.23$} \\
\hline & \multicolumn{2}{|c|}{ Univariate } & \multicolumn{2}{|c|}{ Multivariate } & \multicolumn{2}{|c|}{ Univariate } & \multicolumn{2}{|c|}{ Multivariate } \\
\hline & $\mathrm{HR}$ & $P$ & $\mathrm{HR}$ & $P$ & $\mathrm{HR}$ & $P$ & $\mathrm{HR}$ & $P$ \\
\hline Age, years & 1.010 & 0.285 & & & 1.017 & 0.301 & & \\
\hline \multicolumn{9}{|l|}{ Gender } \\
\hline Male & Ref. & & & & Ref. & & & \\
\hline Female & 1.354 & 0.145 & & & 0.757 & 0.414 & & \\
\hline \multicolumn{9}{|l|}{ Race } \\
\hline White & Ref. & & & & Ref. & & & \\
\hline Others & 1.041 & 0.839 & & & 1.254 & 0.469 & & \\
\hline \multicolumn{9}{|l|}{ Marital status } \\
\hline Unmarried & Ref. & & & & Ref. & & & \\
\hline Married & 0.794 & 0.143 & & & 1.153 & 0.602 & & \\
\hline \multicolumn{9}{|l|}{ Primary site } \\
\hline Pyriform sinus & Ref. & & & & Ref. & & & \\
\hline Others & 1.004 & 0.981 & & & 1.307 & 0.364 & & \\
\hline Tumor size, cm & 1.014 & 0.001 & 1.011 & 0.049 & 1.018 & 0.047 & 1.016 & 0.068 \\
\hline \multicolumn{9}{|l|}{ Tumor grade } \\
\hline $\mathrm{G} 1+\mathrm{G} 2$ & Ref. & & & & Ref. & & & \\
\hline $\mathrm{G} 3+\mathrm{G} 4$ & 0.926 & 0.625 & & & 0.769 & 0.338 & & \\
\hline \multicolumn{9}{|l|}{ T stage } \\
\hline T1-T2 & Ref. & & Ref. & & Ref. & & & \\
\hline T3-T4 & 1.510 & 0.019 & 1.254 & 0.316 & 1.642 & 0.124 & & \\
\hline \multicolumn{9}{|l|}{ N stage } \\
\hline N1 & Ref. & & & & NA & NA & NA & NA \\
\hline N2 & 1.393 & 0.087 & & & Ref. & & Ref. & \\
\hline N3 & 1.268 & 0.622 & & & 2.201 & 0.022 & 1.931 & 0.083 \\
\hline \multicolumn{9}{|l|}{ M stage } \\
\hline MO & Ref. & & Ref. & & Ref. & & & \\
\hline M1 & 3.756 & 0.010 & 2.995 & 0.062 & 1.261 & 0.699 & & \\
\hline \multicolumn{9}{|l|}{ Surgical type } \\
\hline Local tumor resection & Ref. & & & & Ref. & & & \\
\hline Pharyngectomy & 1.136 & 0.687 & & & 1.005 & 0.993 & & \\
\hline Pharyngectomy with laryngectomy & 1.457 & 0.118 & & & 1.790 & 0.160 & & \\
\hline \multicolumn{9}{|l|}{ POAT } \\
\hline Without & Ref. & & & & Ref. & & Ref. & \\
\hline With & 0.795 & 0.240 & & & 0.423 & 0.006 & 0.489 & 0.037 \\
\hline
\end{tabular}

HR, hazard ratio; LNR, lymph node ratio; NA, not available; POAT, postoperative adjuvant therapy. 
Table S2 Univariable and multivariable Cox regression model for predictors of overall survival in patients with LNR $<0.23$ and LNR $\geq 0.23$

\begin{tabular}{|c|c|c|c|c|c|c|c|c|}
\hline \multirow{3}{*}{ Variable } & \multicolumn{4}{|c|}{ LNR $<0.23$} & \multicolumn{4}{|c|}{$L N R \geq 0.23$} \\
\hline & \multicolumn{2}{|c|}{ Univariate } & \multicolumn{2}{|c|}{ Multivariate } & \multicolumn{2}{|c|}{ Univariate } & \multicolumn{2}{|c|}{ Multivariate } \\
\hline & $\mathrm{HR}$ & $\mathrm{P}$ & HR & $\mathrm{P}$ & $\mathrm{HR}$ & $\mathrm{P}$ & $\mathrm{HR}$ & $\mathrm{P}$ \\
\hline Age, years & 1.016 & 0.046 & 1.019 & 0.019 & 1.028 & 0.073 & & \\
\hline \multicolumn{9}{|l|}{ Gender } \\
\hline Male & Ref. & & & & Ref. & & & \\
\hline Female & 1.250 & 0.220 & & & 0.846 & 0.585 & & \\
\hline \multicolumn{9}{|l|}{ Race } \\
\hline White & Ref. & & & & Ref. & & & \\
\hline Others & 1.077 & 0.659 & & & 1.350 & 0.293 & & \\
\hline \multicolumn{9}{|l|}{ Marital status } \\
\hline Unmarried & Ref. & & & & Ref. & & & \\
\hline Married & 0.789 & 0.077 & & & 0.990 & 0.967 & & \\
\hline \multicolumn{9}{|l|}{ Primary site } \\
\hline Pyriform sinus & Ref. & & & & Ref. & & & \\
\hline Others & 0.988 & 0.928 & & & 1.240 & 0.437 & & \\
\hline Tumor size, cm & 1.011 & 0.004 & 1.012 & 0.003 & 1.018 & 0.037 & 1.015 & 0.075 \\
\hline \multicolumn{9}{|l|}{ Tumor grade } \\
\hline $\mathrm{G} 1+\mathrm{G} 2$ & Ref. & & & & Ref. & & & \\
\hline $\mathrm{G} 3+\mathrm{G} 4$ & 0.966 & 0.794 & & & 0.733 & 0.220 & & \\
\hline \multicolumn{9}{|l|}{ T stage } \\
\hline $\mathrm{T} 1-\mathrm{T} 2$ & Ref. & & & & Ref. & & & \\
\hline T3-T4 & 1.299 & 0.068 & & & 1.422 & 0.220 & & \\
\hline \multicolumn{9}{|l|}{ N stage } \\
\hline $\mathrm{N} 1$ & Ref. & & & & NA & NA & & \\
\hline $\mathrm{N} 2$ & 1.195 & 0.261 & & & Ref. & & & \\
\hline N3 & 0.988 & 0.976 & & & 1.853 & 0.068 & & \\
\hline \multicolumn{9}{|l|}{ M stage } \\
\hline MO & Ref. & & Ref. & & Ref. & & & \\
\hline M1 & 3.866 & 0.003 & 3.569 & 0.013 & 1.940 & 0.160 & & \\
\hline \multicolumn{9}{|l|}{ Surgical type } \\
\hline Local tumor resection & Ref. & & & & Ref. & & & \\
\hline Pharyngectomy & 1.284 & 0.334 & & & 0.883 & 0.812 & & \\
\hline Pharyngectomy with laryngectomy & 1.396 & 0.098 & & & 1.911 & 0.094 & & \\
\hline \multicolumn{9}{|l|}{ POAT } \\
\hline Without & Ref. & & & & Ref. & & Ref. & \\
\hline With & 0.761 & 0.096 & & & 0.417 & 0.003 & 0.459 & 0.016 \\
\hline
\end{tabular}

HR, hazard ratio; LNR, lymph node ratio; NA, not available; POAT, postoperative adjuvant therapy. 